\title{
Erratum to: Plasma exchange response in 34 patients with severe optic neuritis
}

\author{
Romain Deschamps ${ }^{1} \cdot$ Antoine Gueguen $^{1} \cdot$ Nathalie Parquet $^{2} \cdot$ Samir Saheb $^{3}$. \\ Francoise Driss $^{4} \cdot$ Malcie Mesnil $^{5} \cdot$ Catherine Vignal $^{6} \cdot$ Jennifer Aboab $^{1}$. \\ Raphael Depaz $^{1} \cdot$ Olivier Gout $^{1}$
}

Published online: 8 June 2017

(c) Springer-Verlag Berlin Heidelberg 2017

\section{Erratum to: J Neurol (2016) 263:883-887 DOI 10.1007/s00415-016-8073-8}

The author would like to correct the errors in the publication of the original article. The corrected details are given below for your reading.

In the Introduction, the word "since" has been omitted. The correct sentence is: "Since a positive cross-over shamcontrolled, use of plasma exchange (PLEX) had been widely accepted..."

In the Table 1:

It is not "Time to MP, median (mean; range) months" but "Time to MP, median (mean; range) days".
It is not "Time to PLEX, median (mean; range), months" but "Time to PLEX, median (mean; range), days".

It is not "Number of PLEX, median (mean; range), months" but "Number of PLEX, median (mean; range)".

It is not "LogMAR VA before PLEX, median (mean; range), days" but "LogMAR VA before PLEX, median (mean; range)".

It is not "LogMAR VA after PLEX, median (mean; range), days" but "LogMAR VA after PLEX, median (mean; range)".

The online version of the original article can be found under doi:10.1007/s00415-016-8073-8.

Romain Deschamps

rdeschamps@fo-rothschild.fr

1 Service de Neurologie, Fondation Ophtalmologique Adolphe de Rothschild, 25 Rue Manin, 75019 Paris, France

2 Unité d'hémaphérèse thérapeutique, Hôpital Saint-Louis, APHP, Paris, France

3 Service d'hématologie clinique et de Thérapie Cellulaire, Hôpital de la Pitié-Salpêtrière, AP-HP, Paris, France

4 Unité de thérapeutique transfusionnelle, Hôpital universitaire Bicêtre, AP-HP, Le Kremlin-Bicêtre, France

5 Département d'anesthésie réanimation, Fondation Ophtalmologique Adolphe de Rothschild, Paris, France

6 Service d'ophtalmologie, Fondation Ophtalmologique Adolphe de Rothschild, Paris, France 